\title{
Percutaneous vertebroplasty as Treatment of Symptomatic Vertebral Hemangioma
}

\author{
Jin Aun Tan*, Juzaily Fekry Leong, Kamalnizat Ibrahim, Azmi Baharudin, Mohd Hisam Muhamad Ariffin \\ and Shaharuddin Abdul Rhani
}

Department of Orthopaedic and Traumatology, University Kebangsaan Malaysia, Malaysia

Received: March 01, 2018; Published: March 14, 2018

*Corresponding author: Shaharuddin Abdul Rhani, Department of Orthopaedic and Traumatology, Hospital University Kebangsaan Malaysia, Kuala Lumpur, Malaysia, Tel: 60391455555; Email: drshahar73@gmail.com

\begin{abstract}
Vertebral hemangioma is a benign vascular lesion composing of new and malformed blood vessels, which occurs in the spine. These tumors are usually asymptomatic and are incidentally diagnosed during magnetic resonance imaging, performed for other purposes. However, patients may present with pain if there is neural arch expansion, vertebral body enlargement or direct compression to the spinal cord or nerve roots. A number of treatments such as radiotherapy and surgery are used in the treatment of symptomatic patients but both methods have been burdened by complications. Percutaneous verterbroplasty, a minimally invasive procedure, has emerged as a treatment of symptomatic vertebral hemangioma with satisfactory results and low complication rates. We present here a case of symptomatic vertebral hemangioma, which was treated with percutaneous vertebroplasty.
\end{abstract}

Keywords: Hemangioma, percutaneous vertebroplasty, cement, polymethyl methacrylate

\section{Introduction}

Vertebral hemangiomas represent a benign, silent lesion, which are usually diagnosed incidentally, during investigations of other conditions. However, these lesions may be symptomatic if there are radiological signs of aggressiveness such as hypo intensity in T1weighted and hyper intensity in T2-weighted MRI images, evidence of paravertebral soft tissue involvement and presence of cortical erosion [1]. We reported a case of a patient with symptomatic vertebral hemangioma undergone successful percutaneous verterbroplasty, which is a minimal invasive procedure with excellent outcome without any complication.

\section{Case Report}

A 43-year-old lady presented with history of several months of mechanical lower back pain, which was aggravated with extension of his back. The pain was localized over the right lower back and was associated with instability pain upon getting up from a lying position. Otherwise, there was no radicular pain over both lower limbs and symptoms of neurologic claudication. No trauma prior to the onset of symptoms was recorded. Clinical examination revealed that the patient had listing to the left side; otherwise, all other spinal and neurological examinations were normal. An MRI (Figure $1 \& 2$ ) revealed a L4 lesion in the right posterior body, which was hypo intense on T1-weighted image and hyper intense on T2- weighted image, extending to the right pedicle, with no sign of cortical defect. There were also bilateral facet joint hypertrophy and a left sided far lateral annular tear of the L4/L5 disc. Overall, this imaging study was suggestive of a vertebral hemangioma with generalized degenerative changes over the affected lumbar vertebrae.

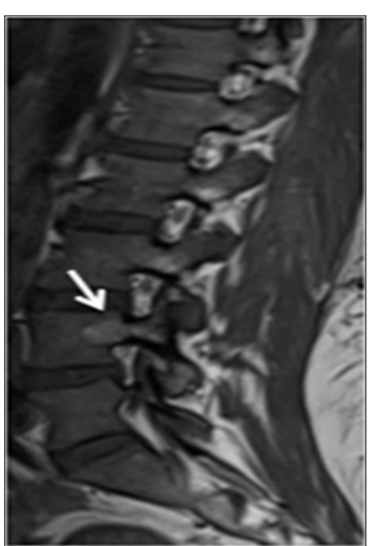

Figure 1: Sagittal T2-Weighted and T1-Weighted Mri Images Showing Hyperintensity Signal, Representing Hemangioma of L4 (Describe the Site of the Lesion). 


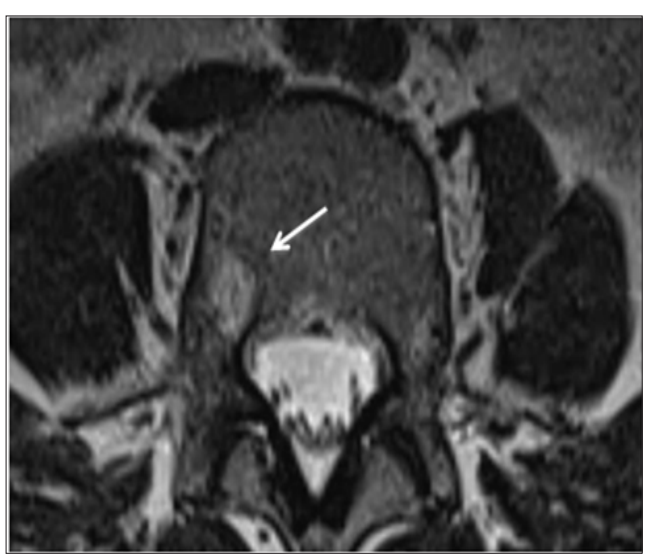

Figure 2: Axial View T2-Weighted Mri Imaging Showing Hyperintensity Signal of Right Vertebral Hemangioma of L4 Body, Extending to the Right Pedicle of L4.

As the symptoms were more to the left side, a trial of treatment with facet block using $7.5 \%$ ropivacaine mixed with triamcinolone was administered to the left facets of the lower lumbar vertebrae. This partially alleviated the pain over the left lower back; however, the instability pain persisted. In view of this, it was concluded that the pain generator was from the right posterior vertebra body and pedicle. The left annular tear and facet joint arthropathy, was due to the offloading effect to the left side. Right percutaneous vertebroplasty was then decided. Right unipedicular vertebroplasty was performed under fluoroscopic guidance. The injection of acrylic bone cement filled the right posterior vertebral body and pedicle of L4 without leakage (Figure 3). The post- operative period was uneventful with immediate resolution of pain allowing the patient to return home 2 days later. The clinical and radiographic outcomes were satisfactory at the follow-up visit, with no significant pain or spinal imbalance observed. Full resumption of previous activities of daily living was also noted.

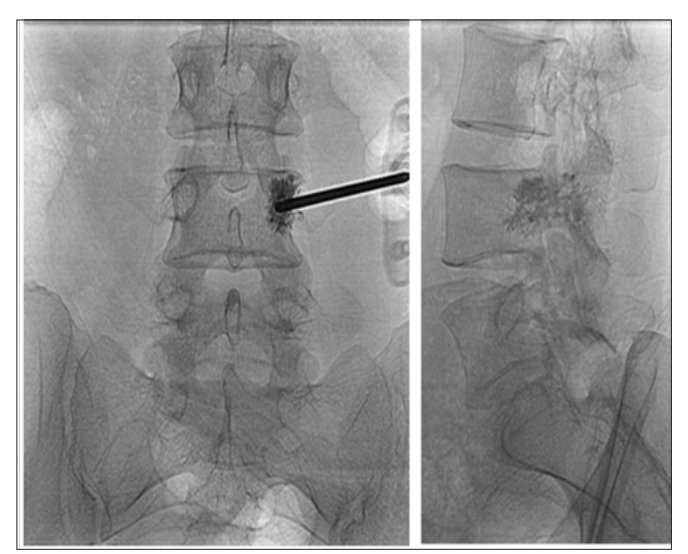

Figure 3: Fluoroscopic View ap and Lateral Showing Needle Position and Radiograph Post Vertebroplasty. Both Views Demonstrated Good Cement Filling.

\section{Discussion}

Patients who are symptomatic should be subjected to a trial of conservative treatment, with brace and oral analgesics for at least 6 weeks before any further treatment is implemented. For those who do not respond to conservative treatment, other options would include surgical decompression, endovascular embolization, radiotherapy and vertebroplasty [2]. Surgical decompression is invasive and may be complicated with excessive hemorrhage during the intra and post-operative periods. In patients with aggressive lesions affecting both the anterior and posterior columns, resection of the affected vertebral would necessitate instrumentation with strut bone grafting. Endovascular embolization is associated with high rates of recurrence and radiotherapy carries a risk of radiation-induced secondary sarcomas or the development of radionecrosis [3]. Vertebroplasty involves administration of polymethyl-methacrylate (PMMA) cement into the vertebral lesion, which will cause irreversible sclerosis of the vessels forming the hemangioma, via its thermal heating effect. The PMMA cement would also infiltrate the cancellous bone of the vertebrae, filling up spaces of micro-fractures, making it more compact. These two actions result in a marked reduction of pain for the patient. Hence, this method has been the treatment of choice as it is minimally invasive, effective in pain reduction, has a low rate of complication, and a shorter length of hospitalization [1].

The main complication associated with vertebroplasty is cement leakage into the prevertebral and paravertebral venous plexus, which may result in spinal cord compression or pulmonary embolism. The risk is due to the presence of disorderly arranged intravertebral vascularization, aggressive lesions, high-flow expansive hemangiomas or formation of intravertebral and paravertebral venous anastomosis. The risk of this complication is reported at $0.5-1 \%$ with good operator skill and the use of high viscosity cement [3]. In the event of cement embolization during the procedure, Narayana et al recommended that the procedure should be abandoned, followed by angio-embolization of the hemangioma, before reattempting vertebroplasty in another setting [2]. Proper patient selection is also important to reduce complications from vertebroplasty. Contraindications include unstable vertebrae fracture with indistinct vertebral collapse, presence of bleeding disorders and infection. An intact cortex of the vertebra is important to prevent cement leakage into any structures around the vertebrae, particularly the spinal canal and foramen. This is less likely in cases of vertebral hemangioma as these lesions do not usually destroy the integrity of the vertebrae as compared to osteoporotic fractures and malignancies.

\section{Conclusion}

Vertebroplasty is a good option of treatment for symptomatic vertebral hemangioma with excellent clinical outcomes. It is a relatively simple procedure which is minimally invasive and has a low rate of complication. Patients should be put under a trial of conservative treatment before undergoing this procedure and proper patient selection is essential to reduce complications rates.

\section{References}

1. Guarnieri G, Ambrosanio G, Vassallo P, Pezzullo MG, Galasso R, et al (2009) Vertebroplasty as treatment of aggressive and symptomatic vertebral hemangiomas : up to 4 years of follow-up. Neuroradiology 51 : 471-476. 
2. Narayana R, Pati R, Dalai S (2014) Percutaneous vertebroplasty in painfu refractory vertebral hemangiomas. Indian Journal of Orthopaedics 48(2): 163-167.

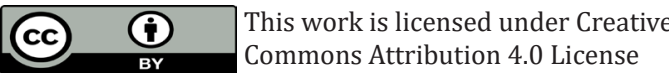

Submission Link: https://biomedres.us/submit-manuscript.php
3. Boschi V, Pogoreli Z, Radoni V, Gulan G, Perko Z, et al. (2011) Management of cement vertebroplasty in the treatment of vertebral hemangioma. Scandinavian Journal of Surgery 100(2): 120-124.

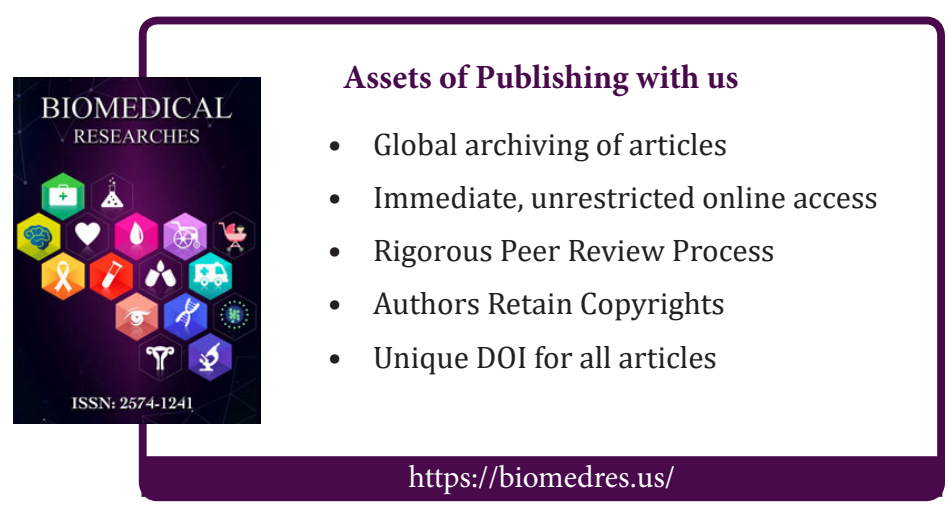

